\title{
MECHANICAL ENERGY AT THE SERVICE OF ECOLOGY
}

When traveling by car, we are generally unaware that shock absorbers are largely responsible for our driving comfort. However, we rather quickly realize when they no longer fulfill their basic purpose, which is to absorb and dissipate the mechanical vibrations generated during our trip. So, what if we changed our approach and instead of dissipating vibrations, tried to use them to increase the energy efficiency of the car?

Scientists from the University of Silesia in Katowice and four other institutions in Spain, Ukraine, Great Britain, and Italy are working on such a concept. The composition of the consortium is completed by the American company Tenneco, the Industrial Partner. The project Electro-Intrusion is funded by the European Union Horizon 2020 program and belongs to the framework of the prestigious FET Proactive competition. The research team at the Silesian University consists of scientists from the Faculty of Science and Technology: Assoc. Prof. Mirosław Chorążewski (head of the project), Dr. Alexander Lowe, and Assoc. Prof. Monika Geppert-Rybczyńska.

The main goal of the project is to create a prototype of an automotive regenerative shock absorber sensitive to mechanical energy (from vibrations occurring while the vehicle is moving) which will transform it, along with thermal energy taken from the environment, into electricity. The conversion will be possible thanks to the phenomena of intrusion and extrusion, which will take place between the non-wetting liquid used in the shock absober and the nanoporous hydrophobic solid.

Imagine a moving car. Its movement will always be accompanied by mechanical vibrations that carry a certain amount of energy which will be absorbed by the suspension of liquid and solid inside the shock absorber. As a result, the liquid will be pushed into the nanoporous solid, i.e., the above-mentioned intrusion will take place. Liquids and solids differ in molecular strucutre and possessing strongly repulsive interactions. Therefore, intrusion requires the supply of mechanical energy, which in the case of our device would be taken from the car's vibrations. After being converted to electricity, this energy would be too small to hold the liquid in the nanopores, so the system would very quickly return to its initial natural state, a process called extrusion. The proper conversion of mechanical energy into electrical energy will be possible thanks to the phenomenon of nanotriboelectrification, in consequence of which an electric charge is generated as a nano-scale friction effect which occurs between the liquid and the solid during successive intrusion and extrusion processes. The electric current generated in this way will power the battery, increasing energy efficiency while driving. Electric cars equipped with regenerative shock absorbers will have an increased range.

"Until now, we have focused on looking for solutions to dissipate energy from car vibrations as efficiently as possible in order to reduce the damage that they can cause continuously," says Dr. Alex- 


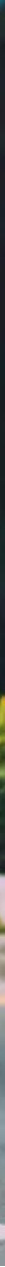

ander Lowe. "What we are working on is a really new idea, an entirely innovative way of thinking about this problem. Since we operate in a nano-scale world, we still do not fully understand all the phenomena occurring in it. Therefore, apart from trying to design a specific device, we also try to thoroughly understand and accurately describe the physical processes that we want to use. Nanotriboelectrification is one of the most difficult processes to understand. The reasons for this are the scale at which it occurs as well as the need for specialized and unique apparatus in connection with computer simulations."

The team of scientists from the University of Silesia analyze the phenomena occurring at the interface between nanoporous solids and liquids, on which the designed shock absorber will be based in the future, in order to determine what materials will be best in this context. It is also important what amounts of mechanical energy (from vibrations) and thermal energy (from the environment) are necessary for the system to work efficiently.

"According to data from the European Environment Agency, a 4 percent drop in total electricity consumption by 2050 can be achieved by installing regenerative shock absorbers in city cars. Our initial estimation is that nanotriboelectric regenerative shock absorbers can reduce fuel consumption by about 9 percent," argues Assoc. Prof. Mirosław Chorążewski.

“The project will last until 2024, and during that time we have a realistic chance to build a working prototype of the shock absorber. Then it will be the task of engineers to transform the results of our research into a product with a final appearance and parameters," Dr. Alexander Lowe sums up.
Assoc. Prof. Mirosław Chorążewski Institute of Chemistry

Faculty of Science and Technology University of Silesia miroslaw.chorazewski@us.edu.pl 